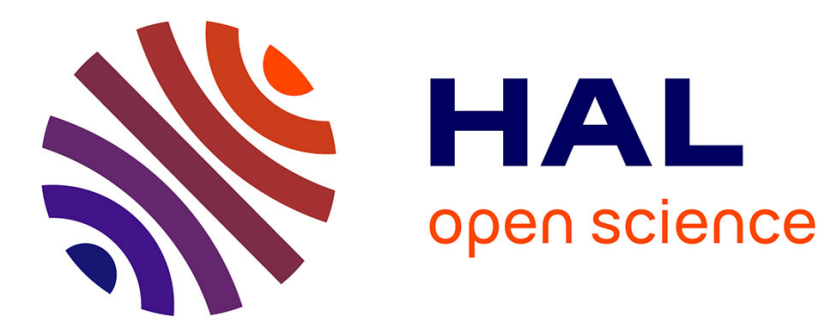

\title{
On Reliable Collaborative Mobility Services
}

\author{
A. Luis Osório, Luis M. Camarinha-Matos, Hamideh Afsarmanesh, Adam \\ Belloum
}

\section{To cite this version:}

A. Luis Osório, Luis M. Camarinha-Matos, Hamideh Afsarmanesh, Adam Belloum. On Reliable Collaborative Mobility Services. 19th Working Conference on Virtual Enterprises (PRO-VE), Sep 2018, Cardiff, United Kingdom. pp.297-311, 10.1007/978-3-319-99127-6_26 . hal-02191195

\section{HAL Id: hal-02191195 \\ https://hal.inria.fr/hal-02191195}

Submitted on 23 Jul 2019

HAL is a multi-disciplinary open access archive for the deposit and dissemination of scientific research documents, whether they are published or not. The documents may come from teaching and research institutions in France or abroad, or from public or private research centers.
L'archive ouverte pluridisciplinaire HAL, est destinée au dépôt et à la diffusion de documents scientifiques de niveau recherche, publiés ou non, émanant des établissements d'enseignement et de recherche français ou étrangers, des laboratoires publics ou privés. 


\title{
On Reliable Collaborative Mobility Services
}

\author{
A. Luis Osório하 Luis M. Camarinha-Matos ${ }^{2}$; Hamideh Afsarmanesh ${ }^{3}$; \\ Adam Belloum ${ }^{3}$ \\ ${ }^{1}$ ISEL - Instituto Superior de Engenharia de Lisboa, Instituto Politécnico de Lisboa, \\ and POLITEC\&ID, Portugal, lo@isel.ipl.pt \\ ${ }^{2}$ Faculty of Sciences and Technology, NOVA University of Lisbon, Portugal, \\ cam@uninova.pt \\ ${ }^{3}$ University of Amsterdam (UvA), The Netherlands \{a.belloum, h.afsarmanesh\}@uva.nl
}

\begin{abstract}
Current approaches for development of collaborative business process automation, when requiring the participation of multiple stakeholders, lack proper formalization in terms of the required informatics systems landscape. Existing solutions depend on specific technology strategies and do not offer a suitable model for the fast-growing collaborative services. In this paper, we present the concept of an open informatics system of systems (ISoS), as a holistic framework that can be applied to a European wide payment system for collaborative multimodal mobility services. To illustrate how the ISoS framework could be applied in practice, we consider the payment service to support public transports, motorway and bridges tolling, payment in parking lots, bicycle renting and payment in a fueling station, all under a single contract. While each participating organization (any infrastructure operator) is free to adopt any applicable technology, the proposed ECoNet collaboration infrastructure is aimed to support a multi-supplier (open) informatics system technology landscape. Based on results from previous research, the paper introduces a strategy to allow effective and reliable EU wide collaborative mobility services.
\end{abstract}

Keywords: Complex Informatics Systems, Collaborative Networks, Collaborative Services, Distributed Systems, Integrated system of systems.

\section{Introduction}

Collaborative services have been gaining importance, namely because of key decisions of the European Commission aiming to push increased competitiveness to enhance value for the European Union. The recent SEPA ${ }^{1}$ directive will make it possible from the beginning of 2018 for any company to offer services based on client's direct debit authorization. This initiative opens the possibility for payment of new collaborative services, offering European citizens a single contract to pay the use of any European mobility-related infrastructure. Such collaborative multimodal mobility services consider payments in any infrastructure, under a single contract and potentially offered by competing providers. This leads to a business scenario in which the European

\footnotetext{
${ }^{1}$ Single euro payments area (SEPA) initiative and direct debit payments legislation, SEPA Direct Debit (SDD)
} 
Citizens are free to select a collaborative mobility services provider (CMSP). An example of a provider can be the Via-Verde company [24], already offering collaborative mobility services in Portugal. The utilization of the infrastructures, e.g. riding a tram, is somehow validated based on mobile technologies [8] (QRCode, NFC/Bluetooth, WiFi), smart cards, biometrics or other authentication/validation mechanisms. To make this scenario clear, we consider that any company acting as a service provider must implements a CMSP reference model, being regulated by mobility authorities. The purpose of a CMSP is to offer collaborative mobility services for citizens, whose payment is made under a unique contract as shown in Figure 1. The mobility authorities or regulators have the responsibility to audit the quality of the offered services and verify if providers are following the contracting policies. Banks provide direct debits from client's accounts, based on explicit debit authorizations. Furthermore, the banks manage the money transfer from a CMSP to the Collaborative Mobility Infrastructure Operators (CMIO) partners (the payments for the utilization of facilities), as direct debits, under the SEPA initiative.

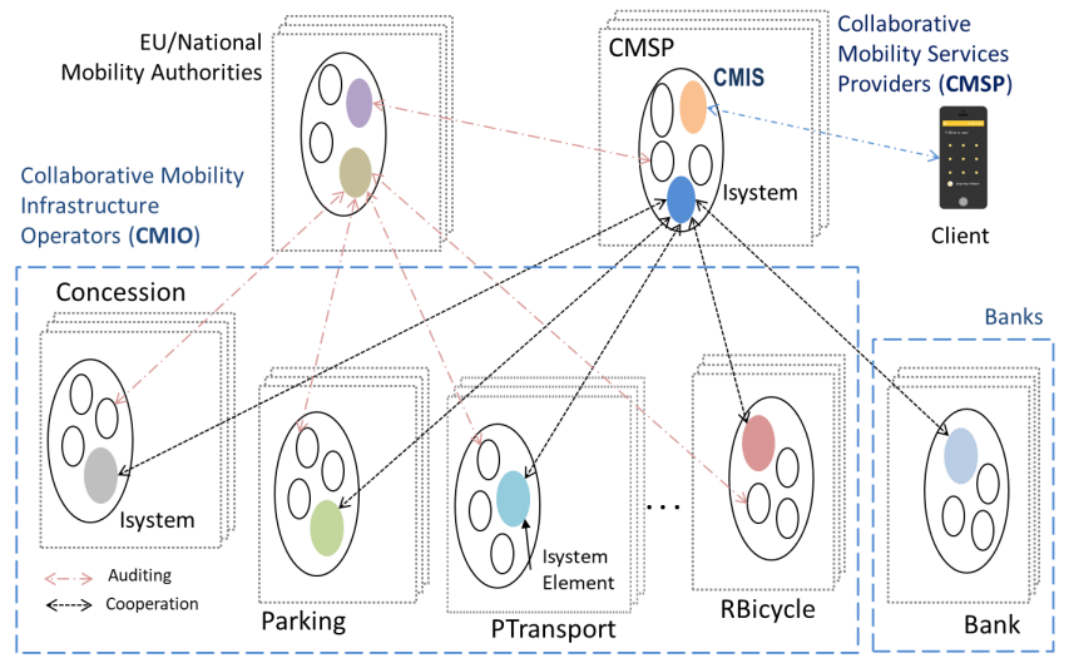

Figure 1 - Collaborative Mobility Services Providers (CMSP)

Handling services offered by more than one stakeholder presents main challenge, since this implies coping with a diversity of processes and technology cultures in need of cooperation [31]. This means that even if citizens (clients) only "see" the multimodal mobility service provider, they in fact, interact with different collaborative mobility infrastructure operators (CMIO) when they travel throughout Europe. This scenario suggests a complex design, operation, and coordination of a composition of services provided by diverse infrastructure operators, banks, mobility regulators and other stakeholders such as call-centers. Consequentely, the CMSP companies face high risks, since the required informatics systems, e.g. the core Collaborative Mobility Informatics System (CMIS), to support billing, invoicing, reporting, analysis and forecasting, customer's assistance, and other functions, face complex integration challenges. Operation and management of the involved computational transactions need to be under an integrated coordination system providing reliable business process automation. 
Additionally, the possibility of scaling up the number of clients and the increasing dependency of businesses from diverse technologies used by the involved parties, make the architecture of a supporting infrastructure, as well as its operational management, complex [3].

In this position paper, the main purpose is to further extend on our previous work in this field, proposing a flexible and scalable infrastructure for an open informatics systems landscape, addressing the CMSP business vision. This infrastructure targets:

i) An open technology architecture where the involved computational elements are substitutable, as a strategy to reduce technology dependencies, and

ii) A unified open collaborative platform, as a strategy to reduce the current complex web of adapters (or mediation systems) linking diverse technology systems of each participating business partner.

The aimed contribution is therefore to propose both, a novel informatics technology infrastructure for CMSP business companies, and a strategy to computationally tie the business and regulator partners under the common collaborative networked organization model [6]. This proposal also assumes a commitment to Collaborative Regulation strategies as discussed in the context of the International Telecommunication Union (ITU) that are established as the 5th generation regulations for sustainable citizen benefit, addressed by the connected digital society [18].

The paper builds on top of previous research on Informatics System of Systems (ISoS) framework [26] and the Cooperative Enabled System (CES) [22] to address reliability in an ambitious validation scenario, and with enhanced requirements. For the Collaborative Networks dimension, we extend the previously proposed Enterprise Collaborative Network (ECoNet) platform [27], contributing for reliability, creation of adaptive ties among partners, and providing a reference implementation of the ISoS framework.

In Section 2, we present the state of the art related to reliable informatics systems architecture and development strategies for collaborative networks. In the Section 3 we discuss the proposed Collaborative Mobility Services Provider scenario for the application of the ISoS model, and the ECoNet framework for structuring the cooperation among informatics systems of the participating stakeholders in order to offer collaborative mobility services. Discussing the conclusions and further research in Section 4 we presents the created value and lessons learned.

\section{State of Research and Trends}

In this sector, we are still in the era of "islands of automation" where the huge standardization efforts made along the last decades remain insufficient [26]. It is interesting to analyze the strategy that has been followed by well-known companies like Amazon, Google, Facebook, LinkedIn, Netflix, that have grown fast on the web and are today the main contributors to interesting open source developments. This strategy seems adequate to induce novel approaches in the market (competition under unified approaches), aiming to reduce dependencies and costs. The highly reliable Apache Kafka messaging system, originally developed by LinkedIn and later incubated as an Apache project [14], [34], is an example of such dynamics. Moreover, there is an 
interesting discussion about dependencies, based on an empirical look at Amacon.com that points to the potential weaknesses of complementing business partners which use platforms to ground their businesses [36]. The conclusion identifies the platform leaders as those active on finding added value, and making partners appear to depend on such sharing of the distribution network, e.g. for the case of Amazon online shop. The above work also mentions situations related to the sharing of technology platforms (Microsoft/Netscape or Apple/App providers) where it seems to be a trend for platform owners to control partners, putting them in a weaker position by indirectly controlling their business. In the purposed scenario, the collaborative mobility services provider is potentially the "powerful node" in the mobility network. Empirical evidence from the Via-Verde case confirms this [24] as well as the development of alternative payment services by members of this network to avoid sharing costs. The Via-Card payment system developed by one of the Portuguese concessionaires, in competition with the Via-Verde service, demonstrates that the management of value in such collaborative networks is a complex endeavor, founded on discussions centered around the risks of collaboration [17].

Furthermore, there is also a recurrent discussion about vendor lock-in regarding the dependencies from cloud service providers [2]. For instance, the mOSAIC system includes an open API to expose what the authors reference as the application (informatics system), making it independent of a specific cloud provider. They further research on common services like discovery, brokering, matchmaking, interoperability, and compositions of services from multiple cloud providers. While challenging, the approach seems to weaken the cloud customers' side, by lacking an analysis of how complex informatics systems shall be structured. In fact, from an interesting survey of web related technologies [15], the main question to address is how to make reliable complex distributed systems, made of existing and upcoming technology diversity, and from the rapidly evolving science and technology. Such fast-evolving dynamics makes it difficult to establish a vendor-agnostic strategy [26]. The interesting survey in [29] identifies several vendor lock-in dependencies from acquisitions by the Information Technology department of the Dutch Tax and Customs Administration (DTCA). A partnership with the suppliers is proposed as a strategy to reduce dependency, in the research classified as a monopoly. These suggestions are aligned with another report on next-generation IT operating models, where collaboration with suppliers is also suggested [20]. The proposed approach is interesting, but in our understanding of the problem it requires more than a change in the relationship between customers and suppliers. The complexity of developing integrated distributed informatics system requires a novel modularity abstraction able to manage the existing tensions among collaborating stakeholders and their specific technology systems that is supporting critical business processes through complex critical systems.

As discussed in [26], existing standards have proved insufficient for an open informatics system. In spite of the importance of the service-oriented architecture (SOA) paradigm for system development, a composition of software and cyberphysical parts into a consistent modular construct, is lacking. The open OSGi specification with its modularity framework and declarative and remote services specifications [9] while important is only one among many de facto adopted specifications. A more recent trend in some approaches involves micro-services. In a report from NIST, a microservice is defined as a "... basic element that results from the 
architectural decomposition of an application's components into loosely coupled patterns consisting of self-contained services that communicate with each other using a standard communications protocol and a set of well-defined APIs, independent of any vendor, product or technology" [13]. This is in fact an architectural style sharing most of the motivations of the SOA principles. One exception however is for the vendor independence, meaning that technology dependencies are the main motivation for this more recent rebranding of service-oriented modularity. In fact, and as far as the business processes, services, and governance models viewpoints are concerned, there is a need to apply a modularity strategy to reduce technology dependencies. In spite of contributions from the ITIL standard, as a structuring and coordination strategy for IT resources, and COBIT, as a reference governance framework for the IT, the multivendor aspect remains an open issue, as discussed in [35]. The question is how to formalize complex technology system setups? The approach is not exclusive to the structuring of software parts that implement services of a system. It is more on how minimal (atomic) they are as computational responsibilities, potentially with multiple suppliers, and how they are composed to establish systems, which in our research are addressed as informatics systems (Isystems) or software-centric systems [26].

An interesting comparison of governance/coordination strategies adopted by SOA and micro-services ( $\mu$ services) is discussed in [7], positioning SOA towards a centralized orchestration and $\mu$ services towards a distributed choreographic model. One problem, however, is the diversity of semantics as an obstacle for a sustainable lifecycle management of complex informatics systems. Our understanding is that the differences among SOA and $\mu$ services are not so relevant to our addressed challenge. The challenge is how to name and structure, with a precise semantics (developing a formal model), the growing complexity of informatics system under a coordinated collaborative multisupplier network of providers of sub-systems, as well as their development, maintenance, and operations services.

Fault tolerance in distributed systems and communication infrastructures is also a key recurrent topic. The Critical Information Infrastructures, for long addressed by the network and distributed systems community, raise the need for robust abstractions. For instance, the statement "we lack a reference architecture of "modern critical information infrastructure'..." in [28] remains a valid point. In fact, no effective formal framework models failures in a collaborative networked organization informatics infrastructure. The question is, how to guarantee that no client has ever to face a failure situation preventing a validation of a payment for a mobility service, somewhere in a public transport in the EU space? For domains involving a large number of distributed elements supporting critical services (as it is the case of the collaborative multimodal mobility services), many of them are dependable from the point of view of the communication infrastructure, operating systems/virtualization, and middleware, as discussed in [10]. Furthermore, the design of fault tolerance mechanisms for reflective middleware components, with modification of the software architecture at runtime, is proposed and discussed in [30]. However, for our discussed problem domain, such a strategy seems difficult to apply, since we address the cooperation among system elements under diverse implementations, different models and technologies, and operations and management responsibilities. 


\section{The Proposed Approach}

The proposed CMSP business case is a challenging scenario. For this context, our approach considers elementary computational responsibilities as parts of an informatics system (commonly referred as software elements or software systems). By system's definition, each element can itself be an informatics system (Isystem), following a decomposition of complexity till manageable elements. One question is what these final atomic elements should be. In our model, they are the Cooperation Enabled Systems (CES) [22]. These atomic elements, viewed as services in an SOA perspective, can be considered as $\mu$ services since they model and operationalize a suite of multi-technology computational services. According to our model, a CES is always under the responsibility and is part of an informatics system (Isystem). The model does not restrict a CES as an informatics system since, according to the ISoS model [26], an informatics system can have a single CES, the CES 0 responsible for supporting the Isystem concept. As discussed in the mentioned paper, the informatics technology landscape of an organization made of Isystems (Isystem, Isystem $_{1}$, Isystem $2, \ldots$, Isystem $_{n}$; for $n>0$ ), includes a meta-Isystem ${ }_{0}$ with the responsibility of governance of the overall technology landscape. Under this model, any Isystem must cooperate with the Isystem ${ }_{0}$ of the organization where it is being deployed to get access to all existing services it is supposed to use (integrate with; dynamic adaptation/interoperation). As a simple example, let us consider users' profiles to manage user's authentication and access rights implemented by a single Isystem $\mathrm{m}_{\mathrm{i}}$. Therefore, we propose the development of further research efforts, answering the requirements of the CMSP scenario, adopting both ISoS and the Enterprise Collaborative platform (ECoNet) [27] as an open specification and open source initiative. The adoption of the ISoS framework and ECoNet with its Enterprise Collaboration Manager (ECoM) Isystem aims at facilitating the adherence of the mobility infrastructure operator, as depicted in Figure 2.

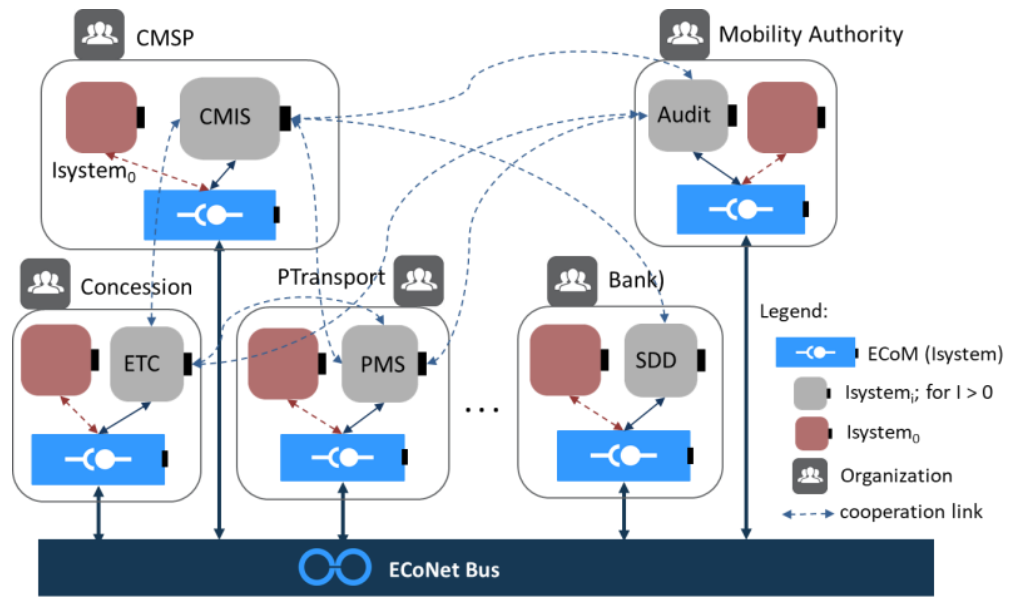

Figure 2 - The CMSP business based on the ECoNet collaborative platform

The adherence of infrastructure operators is one of the main obstacles for the implementation of the CMSP collaborative business. Even if the current approach 
requires the development of specific adapters which are difficult and costly to develop and maintain, this move leads to a perception of the added advantages and openness to the risks of the required changes.

The proposed modeling approach based on both ISoS and ECoNet requires however further research to answer the CMSP requirements. One key issue for the success of the CMMP concept is the ability to scale fast for a complete adherence of infrastructure operators at European level. If the model succeeds, any EU citizen is allowed to access any infrastructure with an offered collaborative multimodal mobility service. On the other hand, in case of success, the CMSP Isystems landscape becomes very complex and critical since a failure without a formal acceptable recovery mechanism can compromise the CMSP business. The ECoNet and ISoS models, based on an open specification and reference implementation, are expected to facilitate the development of both informatics systems and the necessary collaborative contexts (CoC) [23], [27] potentially offered by multiple stakeholders. It is in the interest of CMMP, authorities, and citizens that the business model scales fast under a competing model with the expectation of better services and fair prices.

From the informatics engineering viewpoint, one main contribution is the technology independence and adaptive modularity framework offered by the CES abstraction. However, even if CES is not used to structure an Isystem, a supplier is even able to comply with the ISoS framework. The proposed approach establishes multiple adherence levels with the objective of making easier a shift from the current development culture (legacy) to an effective lower grained multi-supplier model. As suggested in [26], existing informatics systems, e.g., an ERP can wrap the current implementation and offer an equivalent $\mathrm{I}_{0}$ service that is supposed to be implemented by the $\mathrm{CES}_{0}$ element of an Isystem. On the other hand, the legacy ERP can access any other Isystem through the $\mathrm{I}_{0}$ service of the Isystem 0 .

The proposed model further aims to simplify the adoption of coordination mechanisms required to improve reliability. The adoption of Apache Kafka, Zookeeper with its Zab consensus algorithm with ensemble mechanism, and blockchain are research options to introduce reliability by improving fault tolerance and security issues for CES element [37], [1]. The adaptability of CES to multiple implementations (potentially based on different technologies) makes possible for a peer (CES or legacy component) to introspect through the service $\mathrm{I}_{0}$ of a CES element. For an Isystem, the available services are accessed through the $\mathrm{I}_{0}$ service of its $\mathrm{CES}_{0}$, potentially implemented based on different technology frameworks. It means that a CES, as a composite of distributed software elements (threads, objects, procedures/functions, services) can embed more than one execution environment scattered over different physical servers (for fault tolerance), as depicted in Figure 3.

The CES components can be running on-premises on local computer systems or in the cloud. Further research is needed to make CES abstraction redundant by extending the model to support the deployment in a network of computers and this way able to offer fault tolerance capabilities. The approach generalizes an initial restriction to CES elements, making, in this way, possible to develop reliable atoms of an Isystem, as depicted in Figure 3 for a CES component. Furthermore, the Open Adaptive Coupling Infrastructure (OACI) is nothing more than the effective interactions among the multiservices and multi-technology CES elements. In fact, as discussed in [26], through $\mathrm{I}_{0}$ 
any CES or any Isystem through its $\mathrm{CES}_{0}$, can cooperate with any other CES by accessing the required service endpoint.

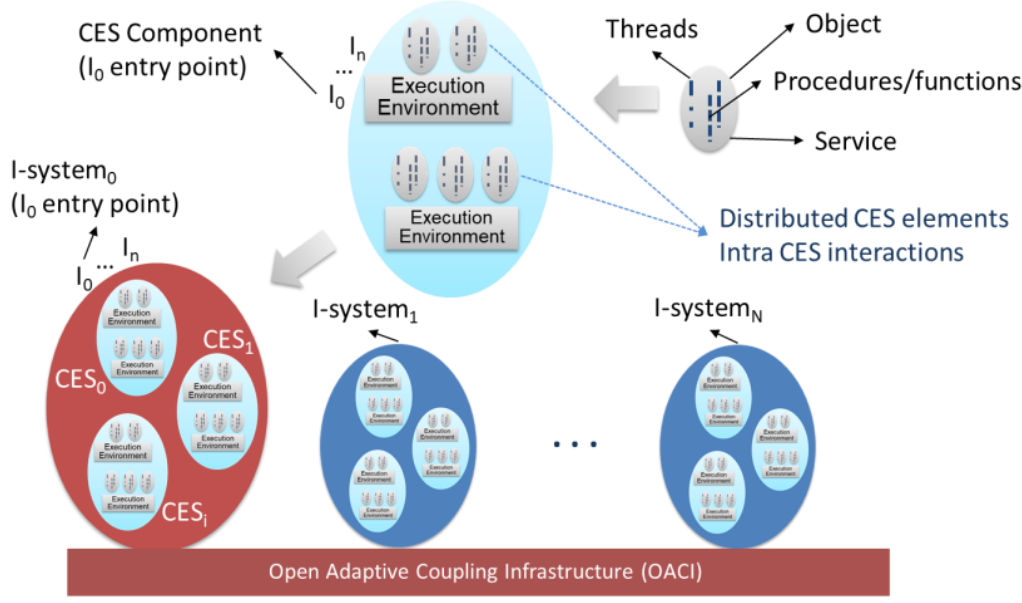

Figure 3 - A detailed view of ISoS and CES elements

Illustrative examples of potential application. The proposed ISoS framework can contribute to accommodate evolving "technology cultures" by encapsulating legacy products into CES abstractions. As an example, and related to the referenced SINCRO project, a cinemometer supplier can make available its product as a CES element by implementing the $\mathrm{I}_{0}$ service, making in this way possible for a peer element to introspect the required interfaces and the supporting technology frameworks. A business challenge like the proposed CMSP needs to assume the state of the art is not ready if competing (multi-supplier) technology solutions are required. The same is happening for several innovative application domains like smart cities, "digital" enterprises (Industry 4.0), digital healthcare, digital public services, where new intelligence services expect to make easier and "green" the citizen's life.

Such holistic endeavors put challenging research questions to the informatics and systems engineering area on how to construct such complex ecosystems under a competition regulated market. Similar to the CMSP scenario, they require a higher or even new integrated approach for the intra-organization domain and reliable networked organization mechanisms for a streamlined collaboration under complex business and technical interactions. The CMSP business is not too different from the logistics single window for a door-to-door freight track, and trace worked in the MIELE project that identified the need of a formal structuration of the networked organizations perspective through the proposed ECoNet platform [27]. It is a step further in understanding how processes, data, computing, and coordination or management models can contribute to an open informatics systems landscape.

The recognized dependencies that are supporting the digital transformation in almost all application domains require sound independent market approaches towards agnostic technology and services provision. The scientific community has here a responsibility, in cooperation with open initiatives, to contribute to realizable 
approaches to be adopted by the market under regulation (conformity certification mechanisms) validation processes. One possibility is to promote tighter coordination between standardization and research efforts towards more open products and services (pluggable into multi-supplier setups). It requires the adoption of promising open specifications and open source led by large user organization, e.g., the interesting case of Google's Kubernetes (embedding Docker) as an interesting framework to manage complex deployments on multiple computing nodes (on-premises or cloud) of reliable informatics systems [32]. It is worthwhile to note that this research only refers the fact that the Kubernetes platform includes an additional rte container engine (beyond docker) suggesting the need for standardization, as the unique threat in the discussed Strengths, Weaknesses, Opportunities, and Threats (SWOT) analysis. If we want the research community to take the lead of a business processes and technology agnostic approach to CMSP and similar challenges, there is a needed for a strategy to induce productization dynamics based on valuable reference implementations, developed under open specifications and open source models. These valuable reference implementations can work as mechanisms (tools) for the scientific foundation of the formulated hypothesis.

\section{Foundations and Value Creation}

One expected added value of this proposal is the possibility (facilitation) for smaller technology and service companies to participate in the development of the upcoming complex informatics systems. The vision of a competitive public procurement based on certified CES elements as building blocks of Isystems is challenging. Its feasibility is partially proven based on the experience with the Portuguese National vehicle speed enforcement network (SINCRO) where suppliers were invited to adhere to a common framework (interfaces) for local cabinets and cinemometers (cyber-physical elements). Some lessons learned. From previous projects showing a clear gap between theoretical models and the real world, it could be observed that value is often not directly viable by a number of reasons like the lack a business driver, the different perceptions of value, etc. For instance, in the case of the ITSIBus model [24], the system concept was never suggested to be adopted by suppliers because of the risk to induce a rise in costs.

The need for higher integration levels and reliable enterprise informatics systems has been for long discussed under the complementary perspectives of business and technology. In some cases, this discussion uses different terms and concepts, depending on the background of the contributors. The case study of the adoption of unified enterprise architecture by the Norwegian higher education sector is an interesting example of the difficulty to establish an integrated business and information and technology vision [21]. It is common research practice to address the automation of business processes to establish specific strategies, e.g., the eRIM framework that proposes an automation strategy for the electronic civil construction requirements [12]. The proposed framework in the mentioned work addresses domain-specific issues based on a SOA architecture but without a clear modularity framework able establish a clear separation between the problem domain and the technology strategy to address it 
under a well-founded model. The need for a new generation of enterprise information systems, guided by an enterprise engineering perspective, is discussed in [11] as a research approach that might take some time to be adopted by the market. A continuous alignment of business and IT is guaranteed by evolving from model-driven engineering to a continuous alignment based on meta-modeling and human-interpretable graphical enterprise architecture and machine-interpretable enterprise ontologies. In this and another paper with common authors, the emphasis is put on enterprise architecture/ontology and the potential of the Zachman's two dimensions enterprise model for the upcoming challenges [16]. This business/IT perspective lacks, however, an analysis of the need to structure the technology landscape aiming to be prepared to answer such modeling and meta-modeling levels under agnostic competitive technology offerings. Furthermore, the model proposed by those authors also lack the collaborative dimension, since organization's technology landscape needs to cope with critical processes at collaborative networks level. We believe that a balanced, holistic approach to business and informatics engineering addressing the complexity of large and distributed informatics system of systems is needed. Based on our research we envisage that enterprise engineering shall evolve to collaborative enterprise engineering under formal constructive models able to scale under dependability in order to ensure a sustainable move to the collaborative digital era. There is a need to consolidate emergent Collaborative Network models like the ARCON reference model [6], [5] towards vendor-agnostic informatics systems for the critical collaborative processes dependent on heterogeneously distributed technology landscapes. The proposed CES, ISoS, ECoNet [25], emerged based on real-world scenarios, contributing to reliable and open informatics systems for collaborative networks, thus potential contributions for the realization of ARCON (meta-modeling framework).

Towards an implementation roadmap. Similarlar to other initiatives, an open source and open specifications initiative needs to be developed to make available validated implementations of ISoS components and a reference implementation of the ECoM with collaborative contexts for the exchange of data and coordination information among $\mathrm{CN}$ members, in addition to other ECoNet platform components. Starting from the prototype developed in the MIELE project, the challenge is to invite informatics systems development companies to support the initiative with the advantage of being them suppliers of supporting products and services. The proposed open innovation model seems to differ from the long-studied open innovation a from company viewpoint [4]. However, the value identified in [33] from the Brisa research investments under an open innovation strategy, make us believe that open source dynamics have the potential to speed-up and scale an effective adoption of the proposed theoretical models. The Brisa company is a highway concessionaire with public responsibility, assuming the investment as a strategy to reduce acquisition costs, reduce the risks to implement new services, and promote product innovation. The strategy is to make available an enhanced version of the ECoMsgExchange reference implementation as a version downloadable through business partner companies with a minimal set of features. The idea is to challenge partners to use the validation demo to establish a multitenant Virtual Collaboration Context with business partners and share messages and files [27]. The main purpose is to strengthen valuable synergies between user organizations and regulators on promoting open specifications validated through 
reference implementations under open source dynamics, with the participation of product and services companies. From previous experience, it is difficult to manage the perception of value by end-organizations, product and service suppliers and also regulators. However, also from previous projects, we observed that given the perception about the de facto fast trend for business digitalization and the need for total integration, both intra and inter organizations, consensus about open specifications is an imperative trend. The Advanced Metering Infrastructure (AMI) is another interesting research area, questioning the need for common water and environment sensors technology [19] that partially corroborates the proposed strategy.

Therefore, the proposed CMSP and similar holistic scenarios (the trend) put some novel research questions that need an answer before business adopts them, since citizens expect them to ensure their legitimate rights. However, given the fierce pressure from the market to offer new business services and given the panoply of technologies, in many cases without proper coordination, there is a risk for our holistic initiative. The scientific community can play here a leading and mediating role by designing approaches and maintaining a continuous research agenda grounded on a conformity certification role (on behalf of public interests). Such a strategy is needed to achieve a generalized multi-supplier technology and services landscape for the fastcoming digital world, under a competent regulation.

\section{Conclusions and Further Research}

The proposed strategy is a step further towards the construction of a model for the complex informatics systems for collaborative networks contexts. The discussed mobility scenario is challenging enough to illustrate the diversity of paradigms from low-level computational services to the collaborative business processes level, under a holistic approach. The CMSP business operators face a risky business since potentially millions of users depend on the reliability of distributed elements from infrastructures and informatics systems and processes from a diversity of organizations around Europe.

With the proposed research direction, based on a partially validated strategy, we aim to contribute to the feasibility of managing a diversity of issues under common formal models. The proposed strategy considers both the intra-organization and the collaborative networked organization's dimension. For the collaborative dimension a specialized Isystem, developed under an open specification and conformity certification process, is adopted. The ECoM Isystem is responsible for managing the relationships between the organization as a node of a collaborative network and its business partners. The ECoNet system was validated as a pilot in the European MIELE project and is adopted by the proposed collaborative multimodal scenario to formalize data and coordination exchanges among multimodal collaborative mobility services provider business partners.

We identify a number of open challenges at different levels for such complex endeavor. The following four levels are considered: i) infrastructure elements (validators at public transport, vehicle classification, license plate recognition, gate in a parking lot, etc.), ii) organizations' Isystem landscape (commonly referenced as IT), iii) Organization's users and partners business processes and services (also commonly 
referenced as IT), and iv) the collaborative network dimension, managing business relationships, trust, and risks. For each of these dimensions we identify further research challenges:

i. Infrastructure elements - How to guarantee that a citizen always has a mechanism to authenticate and make the payment in any infrastructure, even when without a specific credential (mobile, card) or some element of an Isystem in the validation path fails;

ii. Isystem landscape - How to coordinate the substitution of an Isystem, e.g., the Isystem that manages toll payments;

iii. Processes and services - How to coordinate local specific processes (including changes in local policies) with collaborative commitments, e.g., change of already paid multimodal reservations;

iv. Collaborative network - How to manage business risks among networked stakeholders considering that each member has different investment levels in the quality of processes and technology.

Based on the experience from previous partial results, it is expectable that further consolidation of the proposed models, namely the development of reference implementations under an open source and open specifications communities, will help to understand further weaknesses and help to improve formal models. The proposed CMSP scenario is a quite complex one, requiring further validation and adherence, from both collaborative multimodal service providers (CMSP) and infrastructure operators, aiming to evolve for vendor-agnostic technology landscapes. It is also of paramount importance the adherence of technology providers to discuss and contribute to the proposed models, making them prepared to participate in competitive tenders.

Acknowledgments. This work has been partially supported by Galpgeste and BP Portugal through the research project Horus, by the Administration of the Port of Lisbon and Leixões through the MIELE project, the A-to-Be (Brisa Innovation and Technology), and ANSR (National Road Security Authority) through the SINCRO project. Partial support also from the Center of Technology and Systems - Uninova, and the Portuguese FCT-PEST program UID/EEA/00066/2013.

\section{References}

1. A. Ailijiang, A. Charapko, and M. Demirbas. Consensus in the cloud: Paxos systems demystified. In 2016 25th International Conference on Computer Communication and Networks (ICCCN), pages 1-10, Aug 2016.

2. A. Amato, G. Cretella, B. Di Martino, L. Tasquier, and S. Venticinque. Semantic engine and cloud agency for vendor agnostic retrieval, discovery, and brokering of cloud services. In Asma Al-Saidi, Rudolf Fleischer, Zakaria Maamar, and Omer F. Rana, editors, Intelligent Cloud Computing, pages 8-25, Cham, 2015. Springer International Publishing.

3. A. Balalaie, A. Heydarnoori, and P. Jamshidi. Microservices architecture enables devops: Migration to a cloud-native architecture. IEEE Software, 33(3):42-52, May 2016.

4. Julian Birkinshaw. Reflections on open strategy. Long Range Planning, 50(3):423 - 426, 2017. Open Strategy: Transparency and Inclusion in Strategy Processes. 
5. L. M. Camarinha-Matos, H. Afsarmanesh, E. Ermilova, F. Ferrada, A. Klen, and T. Jarimo. ARCON reference models for collaborative networks. In: Collaborative Networks: Reference Modeling, pp 83-112. Springer US, 2008.

6. L. M. Camarinha-Matos and H. Afsarmanesh. Towards a reference model for collaborative networked organizations, 2006. On reference models for collaborative networked organizations, Int. Journal Production Research, 46(9), May 2008, pp 2453 - 2469.

7. T. Cerny, M. J. Donahoo, and M. Trnka. Contextual understanding of microservice architecture: Current and future directions. SIGAPP Appl. Comput. Rev., 17(4):29-45, January 2018.

8. R. Couto, J. Leal, P. M. Costa, and T. Galvão. Exploring ticketing approaches using mobile technologies: Qr codes, nfc and ble. In 2015 IEEE 18th International Conference on Intelligent Transportation Systems, pages 7-12, Sept 2015.

9. F. Cuadrado, J. C. Dueas, J. L. Ruiz, J. Bermejo, and M. Garcia. An open source platform for the integration of distributed services. In 22nd International Conference on Advanced Information Networking and Applications - Workshops (aina workshops 2008), pages 1422-1427, March 2008.

10. M. Garcia-Valls, A. Casimiro, and H. P. Reiser. A few open problems and solutions for software technologies for dependable distributed systems. Journal of Systems Architecture, $73: 1-5,2017$.

11. K. Hinkelmann, A. Gerber, D. Karagiannis, B. Thoenssen, A. van der Merwe, and R. Woitsch. A new paradigm for the continuous alignment of business and it: Combining enterprise architecture modelling and enterprise ontology. Computers in Industry, 79:77 86, 2016.

12. A. Karim Jallow, P. Demian, C. J. Anumba, and A. N. Baldwin. An enterprise architecture framework for electronic requirements information management. International Journal of Information Management, 37(5):455 - 472, 2017.

13. A. Karmel, R. Chandramouli, and M. Iorga. Nist definition of microservices, application containers and system virtual machines. Technical report, NIST - National Institute of Standsrds and Technology, 2016.

14. J. Kreps, N. Narkhede, and J. Rao. Kafka: A distributed messaging system for log processing. In Proceedings of 6th International Workshop on Networking Meets Databases (NetDB), Athens, Greece, 2011.

15. H. Lampesberger. Technologies for web and cloud service interaction: a survey. Service Oriented Computing and Applications, 10(2):71-110, Jun 2016.

16. J. Lapalme, A. Gerber, A. Van der Merwe, J. Zachman, M. De Vries, and K. Hinkelmann. Exploring the future of enterprise architecture: A zachman perspective. Computers in Industry, 79:103 - 113, 2016.

17. P. Macedo and L. M. Camarinha-Matos. Value systems alignment analysis in collaborative networked organizations management. Applied Sciences, 7(12).

18. S. Maddens. Building blocks for smart societies in a connected world: A regulatory perspective on fifth generation collaborative regulation. Technical report, ITU, Regulatory and Market Environment Division, BDT, 2016.

19. M. P. McHenry. Technical and governance considerations for advanced metering infrastructure/smart meters: Technology, security, uncertainty, costs, benefits, and risks. Energy Policy, 59:834 - 842, 2013.

20. S. Mercer, P. Everson, and T. Cox. The next generation of it operating models -6 key thems for the cios. Technical report, Deloitte, 2014.

21. D. H. Olsen and K. Trelsgard. Enterprise architecture adoption challenges: An exploratory case study of the norwegian higher education sector. Procedia Computer Science, 100:804 - 811, 2016. International Conference on ENTERprise Information Systems/International Conference on Project MANagement/International Conference on Health and Social Care Information Systems and Technologies, CENTERIS/ProjMAN / HCist 2016. 
22. A. Osorio, L. M. Camarinha-Matos, and H. Afsarmanesh. Cooperation enabled systems for collaborative networks. In: Adaptation and Value Creating Collaborative Networks, volume 362 of IFIP Advances in Information and Communication Technology, pages 400409. Springer Boston, 2011. 10.1007/978-3-642-23330-2_44.

23. A.L. Osorio, L. M. Camarinha-Matos, and H. Afsarmanesh. Enterprise collaboration network for transport and logistics services. In: Collaborative Systems for Reindustrialization, IFIP Advances in Information and Communication Technology. Springer Berlin, 2013.

24. A.L. Osorio, C. Goncalves, P. Araujo, M. Barata, J. Gomes, G. Jacquet, and R. Dias. Open multi-technology service oriented architecture for its business models: The itsibus etoll services. In: Collaborative Networks and Their Breeding Environments, volume 186 of IFIP International Federation for Information Processing, pages 439-446. Springer Boston, 2005. 10.1007/0-387-29360-4_46.

25. A. L. Osorio. Towards Vendor-Agnostic IT-System of IT-Systems with the CEDE Platform, In: Collaboration in a Hyperconnected World pp 494-505. Springer International Publishing, Cham, 2016.

26. A. L. Osorio, A. Belloum, H. Afsarmanesh, and L. M. Camarinha-Matos. Agnostic Informatics System of Systems: The Open ISoS Services Framework. In: Collaboration in a Data-Rich World pp 407-420, Springer International Publishing, 2017.

27. A.L. Osorio, L. M. Camarinha-Matos, and H. Afsarmanesh. Econet platform for collaborative logistics and transport. In: Risks and Resilience of Collaborative Networks, volume 463 of IFIP Advances in Information and Communication Technology, pages 265276. Springer International Publishing, 2015.

28. P. Verissimo, N. F. Neves, and M. Correia. Crutial: The blueprint of a reference critical information infrastructure architecture. In: Critical Information Infrastructures Security pp 1-14, Springer, 2006.

29. B. Djoerdstra. Dealing with lock-in, http://essay.utwente.nl/70153/1/Sjoerdstra_BA_BMS.pdf, June 2016.

30. M. Stoicescu, J.-C. Fabre, and M. Roy. Architecting resilient computing systems: A component-based approach for adaptive fault tolerance. Journal of Systems Architecture, 73:6 - 16, 2017

31. T. T. Pham Thi, T. L. Dinh, M. Helfert, and M. Leonard. Modelling collaborative services: The COSEMO model. CoRR, abs/1704.03740, 2017.

32. E. Truyen, D. Van Landuyt, V. Reniers, A. Rafique, B. Lagaisse, and W. Joosen. Towards a container-based architecture for multi-tenant saas applications. In Proceedings of the 15th International Workshop on Adaptive and Reflective Middleware, ARM 2016, pages 6:16:6, New York, NY, USA, 2016. ACM.

33. P. Urze and A. Abreu. Knowledge transfer assessment in a co-innovation network. In: Collaborative Networks in the Internet of Services, pages 605-615, Berlin, Heidelberg, 2012. Springer Berlin Heidelberg.

34. G. Wang, J. Koshy, S. Subramanian, K. Paramasivam, M. Zadeh, N. Narkhede, J. Rao, J. Kreps, and J. Stein. Building a replicated logging system with apache kafka. Proc. VLDB Endow., 8(12):1654-1655, August 2015.

35. P. Wiggers, D. Armes, N. Engelhart, and P. McKenzie. SIAM: Principles and Practices for Service Integration and Management. Van Haren Publishing, 2015.

36. F. Zhu and Q. Liu. Competing with complementors: An empirical look at amazon.com. 2015:15468-15468, 01 2015. Harvard Business School Working Paper, No. 15-044, December 2014

37. N. Zupan, K. Zhang, and H.-A. Jacobsen. Hyperpubsub: A decentralized, permissioned, publish/subscribe service using blockchains: Demo. In Proceedings of the 18th ACM/IFIP/USENIX Middleware Conference: Posters and Demos, Middleware '17, pages 15-16, New York, NY, USA, 2017. ACM. 